\title{
A novel, highly discriminatory risk model predicting acute severe right ventricular failure in patients undergoing continuous-flow left ventricular assist device implant
}

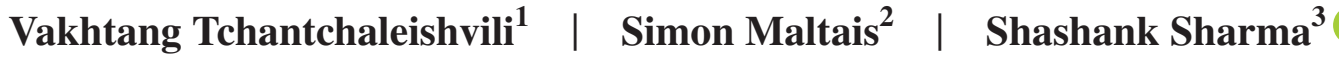 \\ Nicholas A. Haglund ${ }^{4}$ | Mary E. Davis ${ }^{4}$ | Jennifer Cowger ${ }^{5}$ | Palak Shah $^{6}$ | \\ Shashank S. Desai $^{6}$ ｜ Keith D. Aaronson ${ }^{7}$ ｜ Francis D. Pagani ${ }^{7}$ | Shannon M. Dunlay ${ }^{2}$ | \\ John M. Stulak ${ }^{2}$
}

${ }^{1}$ Thomas Jefferson University, Philadelphia, Pennsylvania

${ }^{2}$ Mayo Clinic College of Medicine, Rochester, MN, USA

${ }^{3}$ Cleveland Clinic Foundation, Cleveland, $\mathrm{OH}, \mathrm{USA}$

${ }^{4}$ University of Kansas Medical Center, Kansas City, KS, USA

${ }^{5}$ Henry Ford Medical Center, Detroit, MI, USA

${ }^{6}$ Inova Heart and Vascular Institute, Falls Church, VA, USA

${ }^{7}$ University of Michigan Health System, Ann Arbor, MI, USA

\section{Correspondence}

John M. Stulak, Mayo Clinic College of Medicine, 200 First Street SW, Rochester, MN 55905, USA.

Email: stulak.john@mayo.edu

\begin{abstract}
Various risk models with differing discriminatory power and predictive accuracy have been used to predict right ventricular failure (RVF) after left ventricular assist device (LVAD) placement. There remains an unmet need for a contemporary risk score for continuous flow (CF)-LVADs. We sought to independently validate and compare existing risk models in a large cohort of patients and develop a simple, yet highly predictive risk score for acute, severe RVF. Data from the Mechanical Circulatory Support Research Network (MCSRN) registry, consisting of patients who underwent CF-LVAD implantation, were randomly divided into equal-sized derivation and validation samples. RVF scores were calculated for the entire sample, and the need for a right ventricular assist device (RVAD) was the primary endpoint. Candidate predictors from the derivation sample were subjected to backward stepwise logistic regression until the model with lowest Akaike information criterion value was identified. A risk score was developed based on the identified variables and their respective regression coefficients. Between May 2004 and September 2014, 734 patients underwent implantation of CF-LVADs [HeartMate II LVAD, 76\% $(n=560)$, HeartWare HVAD, 24\% $(n=174)]$. A RVAD was required in $4.5 \%$ $(n=33)$ of the patients [Derivation cohort, $n=15$ (4.3\%); Validation cohort, $n=18$ (5.2\%); $P=0.68)] .19 .5 \%$ of the patients ( $n=143$ ) were female, median age at implant was 59 years (IQR, 49.4-65.3), and median INTERMACS profile was 3 (IQR, $2-3)$. RVAD was required in $4.5 \%(n=33)$ of the patients. Correlates of acute, severe RVF in the final model included heart rate, albumin, BUN, WBC, cardiac index, and TR severity. Areas under the curves (AUC) for most commonly used risk predictors ranged from 0.61 to 0.78 . The AUC for the new model was 0.89 in the derivation and 0.92 in the validation cohort. Proposed risk model provides very high discriminatory power predicting acute severe right ventricular failure and can be reliably applied to patients undergoing placement of contemporary continuous flow left ventricular assist devices.
\end{abstract}




\section{K E Y W O R D S}

left ventricular assist device, right ventricular assist device, right ventricular failure, risk score

\section{1 | INTRODUCTION}

Development of right ventricular failure (RVF) after continuous-flow left ventricular assist device (CF-LVAD) implantation remains a leading cause of perioperative morbidity, end-organ dysfunction, and mortality. ${ }^{1-3}$ Consequently, several risk scores and indices have been reported as useful predictors for the development of RVF following LVAD implantation. ${ }^{1,4-10}$ Most have been developed based on the experience of single institutions and small numbers of patients (Table 1). With improved predictive capabilities, patients at high risk for RVF can be preidentified in the preoperative period and a strategy of short-term support with a temporary right ventricular assist device (RVAD) has been shown to reduce perioperative morbidity, mortality, and reduce hospital length of stay. ${ }^{11}$

However, many risk scores were derived from populations treated with pulsatile LVADs that are no longer used in clinical practice and the outcome of RVF is variably defined. There is currently no RVF risk score that is uniformly accepted. As such, there is a need for developing and validating a contemporary risk score in a large multicenter patient cohort that focuses on current-generation CF-LVADs.

\section{2 | METHODS}

A collaborative multi-institutional retrospective analysis of all primary CF-LVAD implanted as part of the Mechanical Circulatory Support Research Network (MCSRN) database was conducted. MCSRN is a prospectively collected retrospective database run by a dedicated long-term data manager using REDCap platform, who coordinated data entry at each member site. At the time of this project's performance, MCRN consisted of data from Mayo Clinic, University of Michigan, and Vanderbilt University. Acute severe RV failure was defined as need for RVAD support within the index CF-LVAD hospital stay. RVAD support included all temporary and durable right-sided devices. Data used as input variables were acquired from patients' preoperative workup, including preoperative laboratory workup, echocardiograms, and cardiac catheterization.

The MCSRN data set was divided randomly into 2 equal samples ( $n=367$ each). The derivation cohort was used for MCSRN RVF risk model development, while a validation cohort was used for its validation. The MCSRN RVF risk score was developed using preimplant data from the derivation cohort. With the goal to maximally utilize the continuous data, dichotomization into categorical variables was avoided when developing the new risk model. Instead, logarithmic data transformation was utilized as needed for continuous data with skewed distribution and highly variable absolute ranges.

Candidate variables for the MCSRN RVF risk score included preoperative patient characteristics and demographics (age, gender, race, height, body mass index, body surface area, heart failure etiology, device indication), comorbidities (atrial fibrillation, hypertension, diabetes, dialysis-dependent renal failure), preoperative clinical status (intubated, intraaortic balloon pump, INTERMACS profile), preoperative laboratory (serum creatinine level, serum total bilirubin, aspartate aminotransferase, alanine aminotransferase, albumin, brain natriuretic peptide, hemoglobin, white blood cell count, platelet count, international normalized ratio), cardiopulmonary hemodynamics (preoperative heart rate, mean arterial pressure, central venous pressure, systolic pulmonary artery pressure, pulmonary capillary wedge pressure, cardiac index), and echocardiographic (left ventricular ejection fraction, left ventricular end diastolic diameter, mitral regurgitation grade, tricuspid regurgitation grade, right ventricular dysfunction severity) variables (For regurgitation severity, $0=$ none, $0.5=$ trace, $1=$ mild, $1.5=$ mild to moderate, $2=$ moderate, $2.5=$ moderate to severe, $3=$ severe).

The MSCRN RVF Risk Score was compared to commonly used RVF predictor scores and indices, including the ones not reported in the literature for this purpose [central venous pressure to pulmonary capillary wedge pressure ratio (CVP/PCWP), model for end-stage liver disease (MELD)]. A nested cohort was used to allow comparative analyses of the predictor models.

\section{1 | Statistical analyses}

$\mathrm{R}$ statistical software, version 3.2.3 ( $\mathrm{R}$ Foundation for Statistical Computing, Vienna, Austria) was used for data analysis and visualization. For continuous variables, median with interquartile range (IQR) was used given significant deviation from normality $(P<0.05$, Shapiro-Wilk test). Categorical data were presented as percentages and analyzed using the chi-square test. Numeric values of the severity of valve regurgitation were treated as ranks on the ordinal scale. Continuous variables were compared using Wilcoxon ranksum test, while categorical variables were analyzed using the chi-square test.

Candidate predictors for the MCSRN RVF risk model were entered into the model development process and subjected to backward stepwise logistic regression based on 


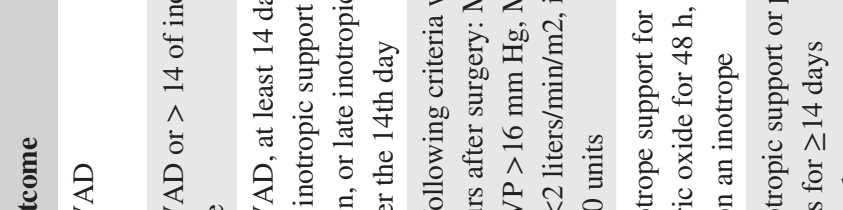

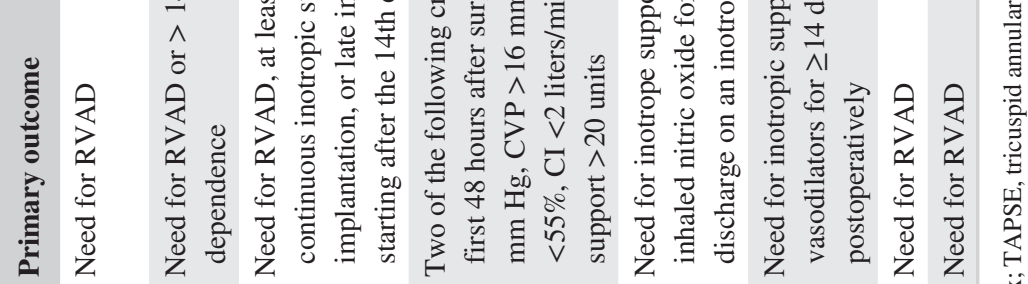

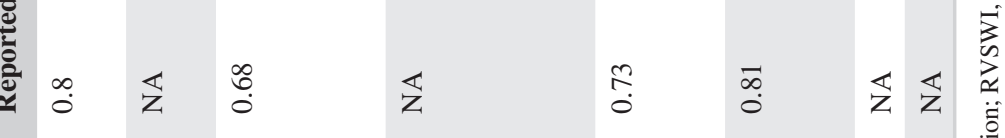

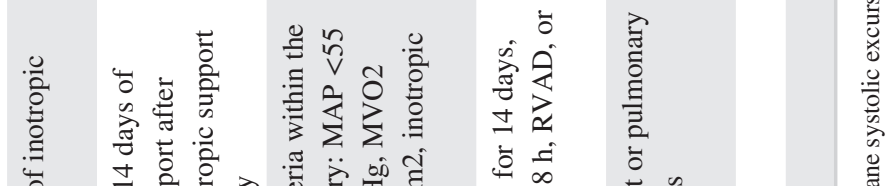

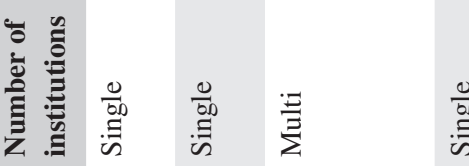

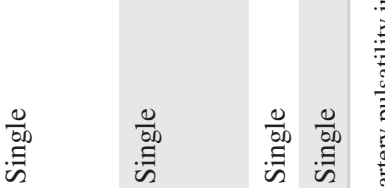

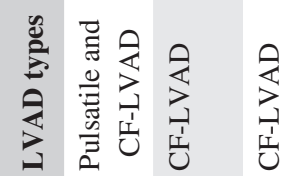

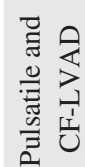

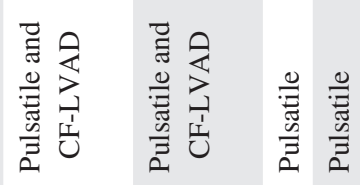

$\div$

范

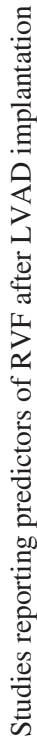

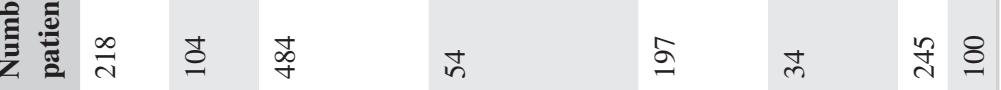

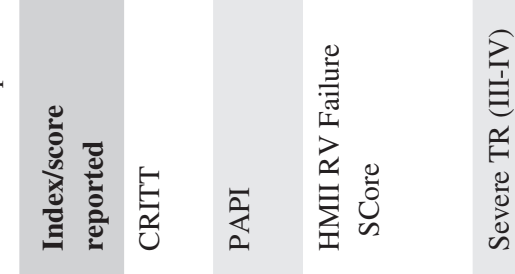

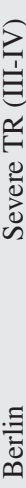

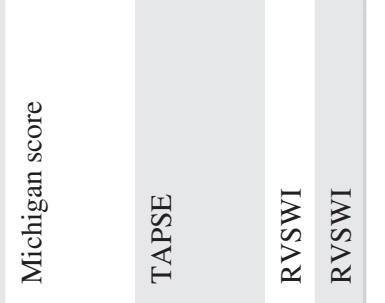

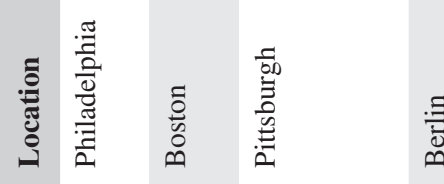

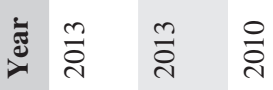

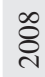

ठั่

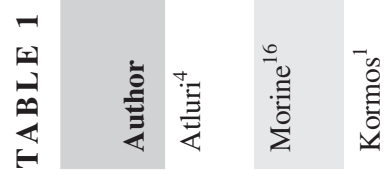

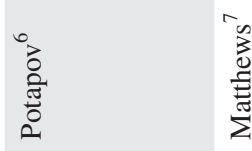

焉

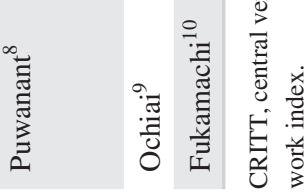


TA B L E 2 Baseline patient characteristics

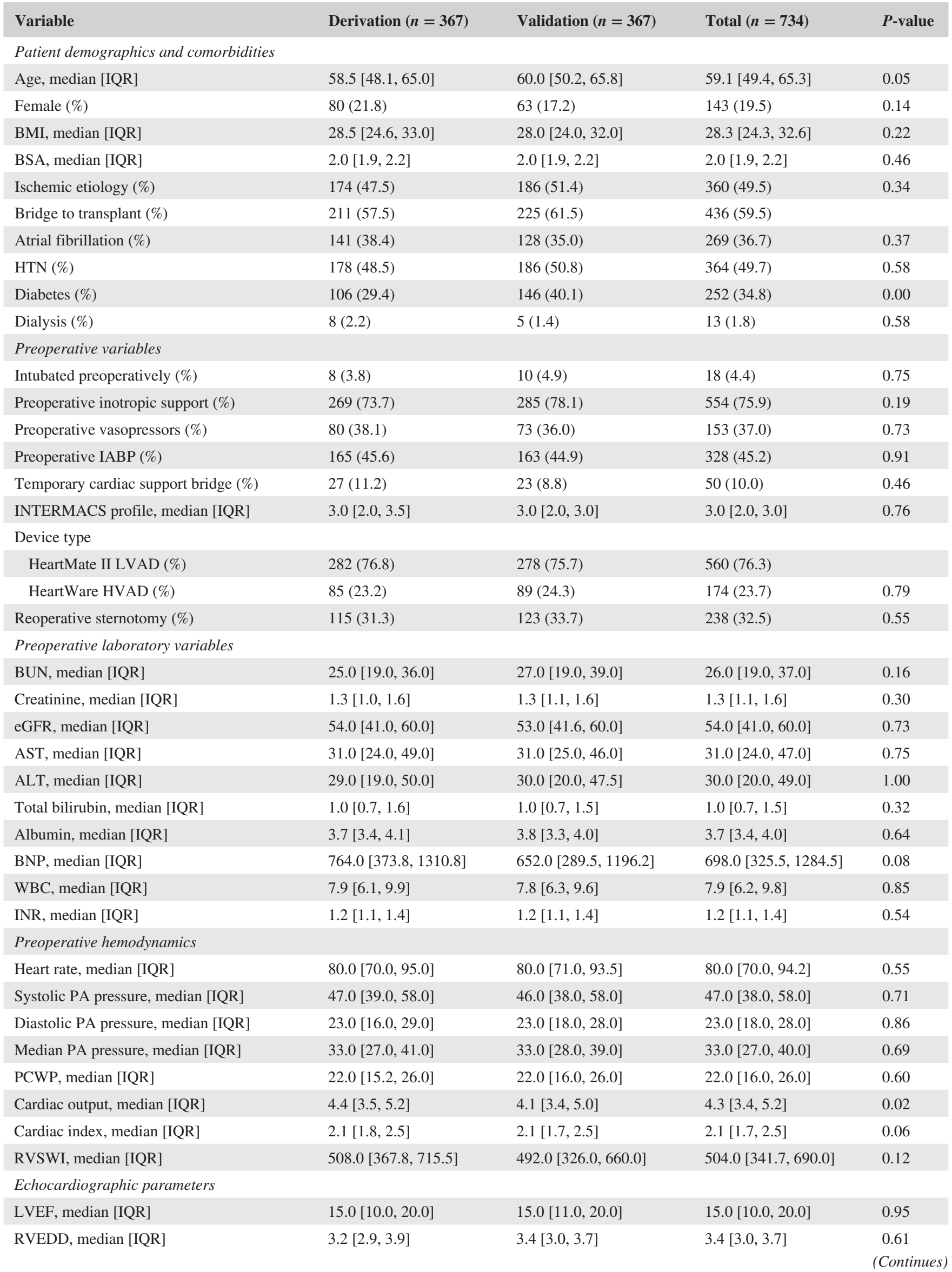


TA B L E 2 (Continued)

\begin{tabular}{|c|c|c|c|c|}
\hline Variable & Derivation $(n=367)$ & Validation $(n=367)$ & Total $(n=734)$ & $P$-value \\
\hline LVESD, median [IQR] & $59.0[8.9,69.0]$ & $58.0[7.8,67.0]$ & $59.0[8.1,68.0]$ & 0.32 \\
\hline Median degree of TR [IQR] & $2.0[1.0,3.0]$ & $2.0[1.0,3.0]$ & $2.0[1.0,3.0]$ & 0.29 \\
\hline Median degree of RV dysfunction [IQR] & $2.0[2.0,3.0]$ & $2.0[2.0,3.0]$ & $2.0[2.0,3.0]$ & 0.80 \\
\hline
\end{tabular}

ALT, alanine aminotransferase; AST, aspartate aminotransferase; BMI, body mass index; BSA, body surface area; BNP, brain natriuretic peptide; BUN, blood urea nitrogen; eGFR, estimated glomerular filtration rate; HTN, hypertension; IABP, intra-aortic balloon pump; INR, international normalized ratio; LVEDD, left ventricular end diastolic dimension; LVEF, left ventricular ejection fraction; LVESD, left ventricular end systolic dimension; MR, mitral regurgitation; PCWP, pulmonary capillary wedge pressure; RVEDD, right ventricular end diastolic dimension; RVSWI, right ventricular stroke work index; TR, tricuspid regurgitation; WBC, white blood cell count.

Akaike Information Criterion (AIC). ${ }^{12}$ First, highly correlated data in the same category (eg, systolic, diastolic, and mean PA pressure values) were subjected to regression based on the lowest AIC value to eliminate all but most promising variables prior to entering them into the model development process. Baseline variables were then entered into the backward stepwise logistic regression process. The baseline variables entered into the analysis are provided in Table 2 . Each time, 8-12 variables were entered into the stepwise regression process to avoid overfitting the model. The lowest AIC level was used to identify the best fitting model. Calibration of the model was assessed using the HosmerLemeshow goodness of fit test. Odds of RVF development were assessed in a continuous manner using restricted cubic splines analysis.

Based on the variables in the final model and their respective regression coefficients developed from the derivation cohort, a novel risk score termed the MCSRN RVF risk score was developed. The risk score was tested on the validation cohort to assess its validity and calibration.

Receiver operating characteristic (ROC) analysis was used to derive areas under the curve (AUC) for assessing the discriminatory power of the risk models (including the MCSRN RVF risk model), and to identify the optimal cutoff level between sensitivity and specificity of the novel risk score. DeLong's test ${ }^{13}$ was used for statistical comparison of ROC curves.

For all analysis (except where mentioned), a $P<0.05$ was considered statistically significant. Local IRB approval was granted for data analysis as part of the MCSRN.

\section{3 | RESULTS}

Between May 2004 and September 2014, 734 patients underwent implantation of CF-LVADs [HeartMate II LVAD, 76\% $(n=560)$, HeartWare HVAD, 24\% $(n=174)] .19 .5 \%$ of the patients $(n=143)$ were female, median age at implant was
59 years (IQR, 49.4-65.3), and median INTERMACS profile was 3 (IQR, 2-3). RVAD was required in 4.5\% $(n=33)$ of the patients [Derivation sample, $n=15$ (4.3\%); validation sample, $n=18$ (5.2\%); $P=0.68]$. Patient demographics and baseline clinical characteristics for each group as well as the entire sample are shown in Table 2.

\section{1 | Derivation of the MCSRN RVF risk score}

The candidate variables were entered into the multivariable regression. The following variables were identified as correlates of RVF in the derivation cohort: heart rate, WBC count, albumin level, BUN level, cardiac index, and the numeric value of the severity of the tricuspid valve's regurgitation (Table 3). Using regression coefficients in the final model as respective weights of these variables, the following formula was created to provide the numeric risk score (please refer to the online supplement for the Excel-based risk score calculator):

$$
\begin{aligned}
& 4.2944 \times \log (\mathrm{HR})-4.4917 \times \log (\text { Albumin }) \\
& +1.2029 \times \log (\mathrm{BUN})+1.0599 \times \log (\mathrm{WBC}) \\
& -1.0364 \times \log (\mathrm{CI})+0.8213 \times \text { numeric TR severity }
\end{aligned}
$$

The numeric values of the MCSRN RVF risk model ranged from 14.5 to 26.9 and were normally distributed $(P=0.2$, Shapiro-Wilk test $)$ with mean value of $20.2 \pm 1.8$. The area under the curve was 0.86 (95\% CI 0.74-0.99) for the derivation cohort and 0.92 (95\% CI $0.85-0.99)$ for the validation cohort indicating very good discriminatory power. The area under the curve for the entire sample was 0.89 (95\% CI 0.82-0.96) (Figure 1). Continuous analysis showed exponential increase of odds ratio of requiring an RVAD with increasing risk score numbers $(P<0.001)$ (Figure 2). The Hosmer-Lemeshow test was consistent with good calibration (derivation sample, $P=0.45$; validation sample, $P=0.57$, entire sample, $P=0.17$ ). 
TA B L E 3 Model derived from the derivation cohort after logarithmic data transformation and backward stepwise logistic regression

\begin{tabular}{|c|c|c|c|c|}
\hline Variable & OR & Lower 95\% CI & Upper 95\% CI & $P$ value \\
\hline $\log$ (Albumin) & 0.01 & 0.0 & 0.4 & 0.01 \\
\hline $\log (\mathrm{WBC})$ & 4.2 & 1.0 & 17.9 & 0.05 \\
\hline $\log (\mathrm{CI})$ & 0.1 & 0.0 & 0.4 & $<0.01$ \\
\hline
\end{tabular}

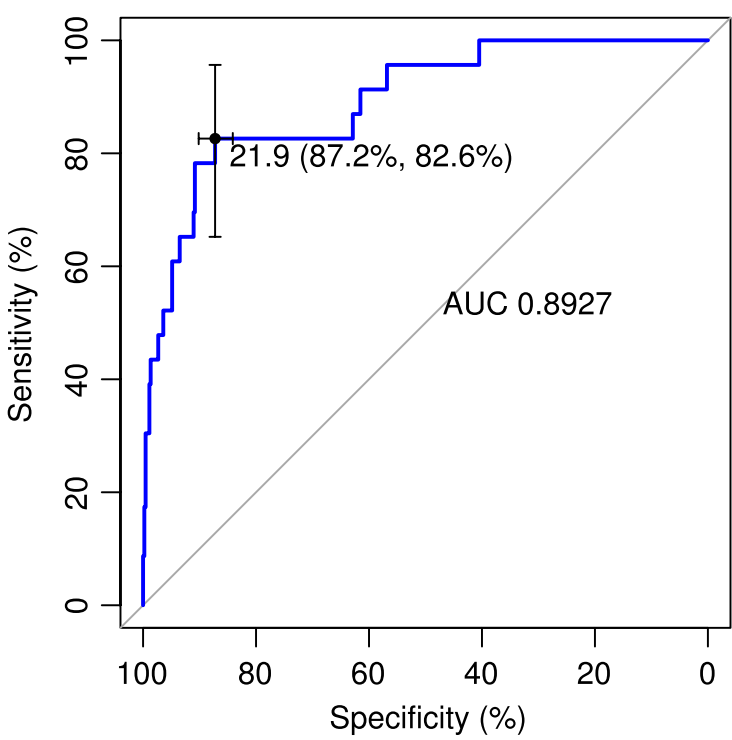

FIG URE 1 Receiver operating characteristic analysis of the novel RV risk model [Color figure can be viewed at wileyonlinelibrary.com]

Based on the ROC analysis of the entire patient sample, the cut-off level of 21.9 yielded the optimal balance between sensitivity (88.4\%) and specificity (78.3\%). The resulting positive predictive value was $98.8 \%$, and the negative predictive value was $25.7 \%$. For clinical simplicity, patients can be stratified into a low-, intermediate-, and high-risk groups using the thresholds 20 and 22, where the incidence of an RVAD was $78 \%$ above the cut-off of 22 , it was $4 \%$ below the cut-off of 20.

\section{2 | Comparison of RVF risk scores}

Areas under the curves for most commonly used RVF risk predictors as calculated by us ranged from poor (AUC $0.60-0.69$ ) to satisfactory (AUC 0.70-0.79): pulmonary artery pulsatility index (PAPI) (AUC 0.78), central venous pressure-RV dysfunction-preoperative intubation-severe tricuspid regurgitation-tachycardia (CRITT) (AUC $0.74)$, right ventricle to left ventricle $(\mathrm{RV} / \mathrm{LV})$ ratio (AUC 0.71), RV stroke work index (RVSWI) (AUC 0.71),

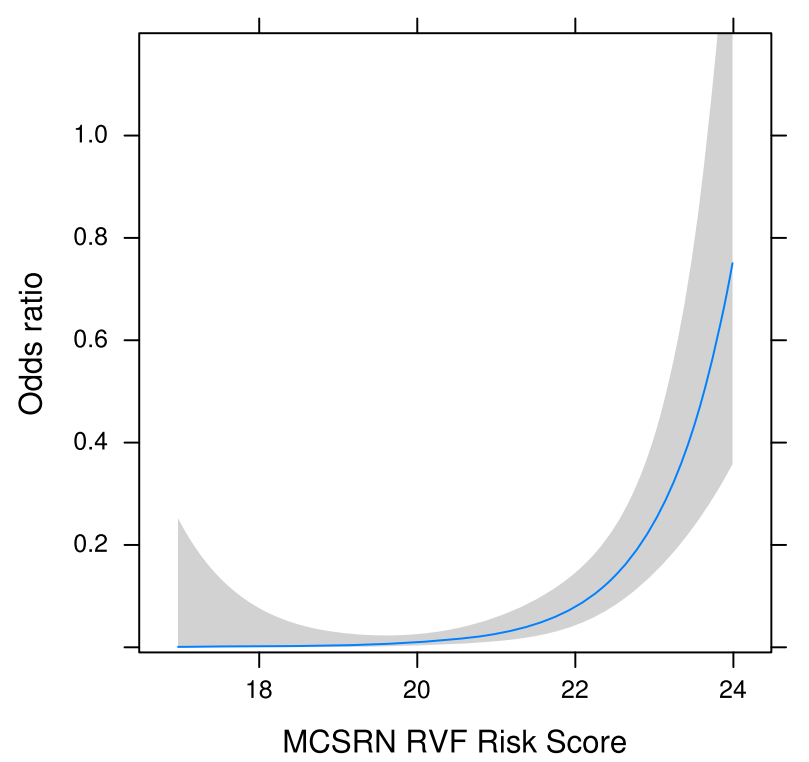

F I G U RE 2 Restricted cubic splines analysis showing continuous relationship between the risk model values and the odds ratios of requiring an RVAD. Odds ratio of needing an RVAD approaches 0 with lower values of the risk model [Color figure can be viewed at wileyonlinelibrary.com]

tricuspid annular plane systolic excursion (TAPSE) (AUC 0.70), MELD (AUC 0.69), CVP/PCWP ratio (AUC 0.68), Severe TR (AUC 0.67), HMII RVF score (AUC 0.64), and Michigan score (AUC 0.61). On AUC comparison, the MCSRN RVF risk score performed better than other scores tested (Table 4 and Figure 3).

\section{4 | DISCUSSION}

Out of the commonly used risk models for RVF in CF-LVAD patients, none of them have been developed with a contemporary patient population. We were not able to identify an existing model with strong performance (AUC 0.80-0.89) to predict acute severe RVF requiring RVAD after CF-LVAD implantation. In contrast, the AUC for our risk model reached 0.89 for the entire sample and there was no drop in the AUC from derivation (AUC 0.86) to validation (AUC 0.92) 
TA B L E 4 Receiver operating characteristic analysis of commonly used RVF predictors applied to the full MCSRN data set $(n=734)$ with need for RVAD as a hard endpoint.

\begin{tabular}{|c|c|c|c|}
\hline Predictor & AUC & $98 \%$ CI & $\begin{array}{l}P \text { value compared with } \\
\text { MCSRN RVF Risk Score }\end{array}$ \\
\hline MCSRN RVF & 0.89 & $0.82-0.96$ & - \\
\hline CRITT & 0.74 & $0.60-0.89$ & 0.02 \\
\hline RVSWI & 0.71 & $0.59-0.83$ & $<0.01$ \\
\hline MELD score & 0.69 & $0.60-0.79$ & 0.05 \\
\hline CVP/PCWP ratio & 0.68 & $0.58-0.79$ & $<0.01$ \\
\hline Severe TR & 0.67 & $0.59-0.76$ & $<0.01$ \\
\hline HMII RVF score & 0.64 & $0.48-0.79$ & 0.05 \\
\hline
\end{tabular}

CRITT, central venous pressure-RV dysfunction-preoperative intubation-severe tricuspid regurgitation-tachycardia; MELD, model for end-stage liver disease; PAPI, pulmonary artery pulsatility index; RVSWI, RV stroke work index; TAPSE, tricuspid annular plane systolic excursion.

cohorts, indicating a strong performance. We chose to use a RVAD as the primary outcome due to varying definitions of RVF at our institutions, many of which are dependent on institutional practice variation in management of inotropes or nitric oxide after CF-LVAD as opposed to an RVAD which is only employed in the sickest cohort of patients with acute severe RVF.

Variables identified to predict RV failure after LVAD placement vary widely ${ }^{1,9,10,14,15}$ and reflect the complexity in the multifactorial mechanism of the RV failure onset. While no single best predictor variable has been identified, some form of RV hemodynamics ${ }^{9,10,16}$ or tricuspid valve function ${ }^{6,8}$ surrogates are most commonly used alone , $^{6-8-10,16}$ or as part of a predictive risk score. ${ }^{1,4}$ Additional variables reflective of renal ${ }^{1,7}$ and liver ${ }^{7,17}$ function have commonly been factored in the prediction process, however are not used alone. Heart rate and cardiac index represent hemodynamic variables that are not specific for the RV function and, to a degree, it is surprising to find them in the final model as they can be altered significantly with different interventions. However, other predictive risk models have used these variables as well. ${ }^{4,18} \mathrm{WBC}$ count has also been identified among the variables associated with RV failure in CF-LVAD patients ${ }^{1,4}$ and may be reflective of a systemic component of the disease, or a sicker patient. An important part of our risk model development process that (to the best of our knowledge) has not been employed in deriving other risk models is not dichotomizing continuous data. This, while imposing additional steps (like logarithmic data transformation) on the process, is more likely to retain the predictive strength both in individual variables and the combined model.

While dichotomization of the continuous variables should be avoided, cut-off values for the continuous scale

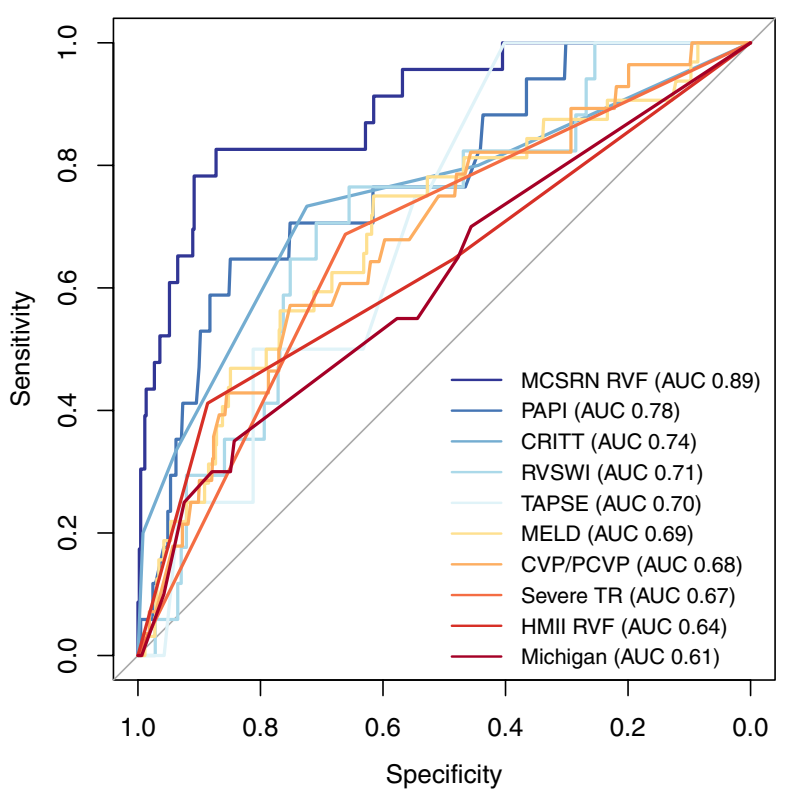

F I G URE 3 Receiver operating characteristic curves illustrating the performance of commonly used RVF predictors. AUC: area under the curve [Color figure can be viewed at wileyonlinelibrary.com]

can be helpful in aiding the physician in the decision process, hence the need for identifying an optimal cut-off that minimizes the compromise between sensitivity and specificity. The cut-off level of approximately 22 resulted in good sensitivity $(88.4 \%)$ and fair specificity $(78.3 \%)$. While the negative predictive value was poor $(25.7 \%)$, the positive predictive value at this cut-off was excellent (98.8\%). Although the negative predictive value was low, the positive predictive value of the model makes it a very useful tool to identify high-risk patients in the preoperative period who would benefit from a temporary or permanent RVAD to mitigate perioperative morbidity and mortality. 
As well, it may be helpful to identify "less ideal" candidates for LVAD, especially those who are being considered DT-LVAD. The numeric value of the model can be of additional use as the further it deviates from the cut-off, the more likely that the outcome becomes positive or negative, further aiding the clinician in decision making.

In medicine, despite many prognostic models that are published each year, relatively few are validated and even fewer find their way into clinical practice. ${ }^{19}$ This is true for RVF risk models as well. Deriving and validating a model on the same data set leads to overly optimistic estimates of the model's accuracy, even if the data set is divided into 2 separate parts for model derivation and validation. ${ }^{19}$ This is because "quirks" individual to a data set will also occur in both the derivation and validation samples that are derived from the same data set and may lead to optimistic estimates of prognostic power. ${ }^{20}$ Therefore, the existing risk scores performed worse using an independent sample set when compared to the data set used for its development. This effect, however, is even more prominent with smaller, single-institution samples that will allow higher variation. Our risk model still needs validation in an external data set.

\section{1 $\quad$ Study limitations}

Even though the analysis was performed using a multi-institutional registry, both patient samples (derivation and validation) stem from the same data and therefore display similar comorbidities and intervention patterns. In addition, there may be similar unidentified confounders in both samples that were not picked up by the analysis and may pose a risk of systemic bias. Because of these reasons, this "split-sample validation" can be overly optimistic and our results should be interpreted with caution.

Our model has 6 variables. A generally recommended number of predictor variables in a model is one variable per 10-20 events. As we only had 15 outcome events, we were not able to follow this recommendation. Otherwise, the low number of RVAD events would not leave us with any model at all. In contrast, the more candidate variables there are in the model, the more opportunity there is for some of those variables to end up in the model purely by chance. This is possible for our model as well; however, we used AIC to mitigate the problem (as AIC rewards for significance while punishing for higher number of predictor variables).

We chose RVAD implant as the endpoint for the risk model as it was a hard endpoint, not influenced by local or institutional practices. One obvious downside is that RVAD is a rare event. With just RVAD as an endpoint, the model may not fully encompass risk stratification of a much larger group of patients with RV failure without the need for RVAD and, as such, may lack in generalizability. In contrast, the strength of the model is that it produces a numeric score where higher values should intuitively mean higher risk for RV failure, even without an RVAD.

In summary, the present model should be considered as an exploratory tool rather than a validated risk score due to low rate of events in both groups. To control for these potential confounders and demonstrate such a generalizability, a rigorous external validation process by other authors is required.

\section{5 | CONCLUSIONS}

To conclude, the proposed RVF MCSRN risk score provides the highest-to-date discriminatory power with an AUC of 0.89 which favors comparably to already published models. The risk score is applicable to contemporary patients implanted with CF-LVADs. After appropriate validation, the risk score may be used to identify patients at very high risk for severe right ventricular failure after LVAD who would benefit from either a temporary or permanent RVAD.

\section{CONFLICT OF INTEREST}

The authors have declared no conflicts of interest for this article.

\section{ORCID}

Shashank Sharma (D) https://orcid.org/0000-0002-8952-4737

\section{REFERENCES}

1. Kormos RL, Teuteberg JJ, Pagani FD, et al. Right ventricular failure in patients with the HeartMate II continuous-flow left ventricular assist device: incidence, risk factors, and effect on outcomes. J Thorac Cardiovasc Surg 2010;139(5):1316-1324.

2. Lazar JF, Swartz MF, Schiralli MP, et al. Survival after left ventricular assist device with and without temporary right ventricular support. Ann Thorac Surg 2013;96(6):2155-2159.

3. Kavarana MN, Pessin-Minsley MS, Urtecho J, et al. Right ventricular dysfunction and organ failure in left ventricular assist device recipients: a continuing problem. Ann Thorac Surg 2002;73(3):745-750.

4. Atluri P, Goldstone AB, Fairman AS, et al. Predicting right ventricular failure in the modern, continuous flow left ventricular assist device era. Ann Thorac Surg 2013;96(3):857-863; discussion 863-864.

5. Vivo RP, Cordero-Reyes AM, Qamar U, et al. Increased right-toleft ventricle diameter ratio is a strong predictor of right ventricular failure after left ventricular assist device. J Heart Lung Transplant Off Publ Int Soc Heart Transplant 2013;32(8):792-799.

6. Potapov EV, Stepanenko A, Dandel M, et al. Tricuspid incompetence and geometry of the right ventricle as predictors of right 
ventricular function after implantation of a left ventricular assist device. J Heart Lung Transplant Off Publ Int Soc Heart Transplant 2008;27(12):1275-1281.

7. Matthews JC, Koelling TM, Pagani FD, Aaronson KD. The right ventricular failure risk score a pre-operative tool for assessing the risk of right ventricular failure in left ventricular assist device candidates. J Am Coll Cardiol 2008;51(22):2163-2172.

8. Puwanant S, Hamilton KK, Klodell CT, et al. Tricuspid annular motion as a predictor of severe right ventricular failure after left ventricular assist device implantation. J Heart Lung Transplant Off Publ Int Soc Heart Transplant 2008;27(10):1102-1107.

9. Ochiai Y, McCarthy PM, Smedira NG, et al. Predictors of severe right ventricular failure after implantable left ventricular assist device insertion: analysis of 245 patients. Circulation 2002;106(12 Suppl 1):I198-I202.

10. Fukamachi K, McCarthy PM, Smedira NG, Vargo RL, Starling $\mathrm{RC}$, Young JB. Preoperative risk factors for right ventricular failure after implantable left ventricular assist device insertion. Ann Thorac Surg 1999;68(6):2181-2184.

11. Anderson MB, Goldstein J, Milano C, et al. Benefits of a novel percutaneous ventricular assist device for right heart failure: the prospective RECOVER RIGHT study of the Impella RP device. J Heart Lung Transplant 2015;34(12):1549-1560. https://doi. org/10.1016/j.healun.2015.08.018.

12. Akaike H. A new look at the statistical model identification. IEEE Trans Autom Control 1974;19(6):716-723. https://doi.org/10.1109/ TAC.1974.1100705.

13. DeLong ER, DeLong DM, Clarke-Pearson DL. Comparing the areas under two or more correlated receiver operating characteristic curves: a nonparametric approach. Biometrics 1988;44:837-845.

14. Raina A, Seetha Rammohan HR, Gertz ZM, Rame JE, Woo YJ, Kirkpatrick JN. Postoperative right ventricular failure after left ventricular assist device placement is predicted by preoperative echocardiographic structural, hemodynamic, and functional parameters. J Card Fail 2013;19(1):16-24. https://doi.org/10.1016/j.cardfail.2012.11.001.
15. Bellavia D, Iacovoni A, Scardulla C, et al. Prediction of right ventricular failure after ventricular assist device implant: systematic review and meta-analysis of observational studies. Eur J Heart Fail 2017;19(7):926-946.

16. Morine K, Kiernan M, Kapur N, Baumgartner R, Pham D, Denofrio D. Pulmonary artery pulsatility index as a novel marker of right ventricular failure following LVAD surgery. J Card Fail 2013;19(8):S17.

17. Bonde $\mathrm{P}, \mathrm{Ku} \mathrm{NC}$, Genovese EA, et al. Model for end-stage liver disease score predicts adverse events related to ventricular assist device therapy. Ann Thorac Surg 2012;93(5):1541-1548.

18. Fitzpatrick JR, Frederick JR, Hsu VM, et al. Risk score derived from pre-operative data analysis predicts the need for biventricular mechanical circulatory support. J Heart Lung Transplant 2008;27(12):1286-1292.

19. Wyatt JC, Altman DG. Commentary: prognostic models: clinically useful or quickly forgotten? BMJ 1995;311(7019):1539-1541.

20. Wade A. Derivation versus validation. Arch Dis Child 2000;83(6):459-460.

\section{SUPPORTING INFORMATION}

Additional supporting information may be found online in the Supporting Information section at the end of the article.

How to cite this article: Tchantchaleishvili V, Maltais $\mathrm{S}$, Sharma $\mathrm{S}$, et al. A novel, highly discriminatory risk model predicting acute severe right ventricular failure in patients undergoing continuous-flow left ventricular assist device implant. Artif Organs. 2019;43:624-632. https://doi.org/10.1111/aor.13413 